\title{
Lateral inhibition: inherent recurrent processes in coherent optical propagation
}

\author{
Minhua Liang
}

\begin{abstract}
Processes that are analogous to the neural process of recurrent lateral inhibition can be found in optical systems that consist of a shift-invariant system and a Fabry-Perot cavity. The properties of the optical recurrent system are derived and demonstrated by computer simulation. The simulation shows that optical lateral inhibition can be used to enhance the outline of an amplitude object and to make phase-only objects directly detectable and visible. The optical recurrent system is compared with frequency-plane spatial filtering. Requirements and practical limitations for the design of an optical recurrent system are also discussed.

Key words: Correlation, diffraction, Fabry-Perot, image enhancement, imaging system, neural networks, optical processing, spatial filtering.
\end{abstract}

\section{Introduction}

Lateral inhibition is an interaction between neurons in which the excitement of one neuron inhibits the excitability of neighboring neurons. These phenomena were originally observed in the retina of the horseshoe crab. Hartline, Ratliff, and Miller have developed a mathematical model in which inhibition is treated as a linear shift-invariant system. ${ }^{1,2}$ The response $r(x)$ of a neuron at position $x$ is composed of the stimulus $s(x)$ of the neuron and the inhibition $i(x)$ supplied by all neurons. This response is expressed as

$$
r(x)=s(x)-i(x)
$$

There are two types of lateral inhibition: (1) nonrecurrent, in which the inhibition term depends explicitly on the stimulus, and (2) recurrent, in which the

\footnotetext{
When this work was performed, the author was with the Department of Electrical Engineering, California Institute of Technology, Pasadena, California 91125. He is now with the Department of Electrical Engineering, University of Louisville, Kentucky 40292. His permanent address is the Shanghai Institute of Optics and Fine Mechanics, Academia Sinica, Post Office Box 800-211, Shanghai 201800, China.

Received 20 November 1992; revised manuscript received 25 June 1993.

0003-6935/94/020158-04\$06.00/0.

() 1994 Optical Society of America.
}

inhibition term depends on the response. The nonrecurrent system is described as

$$
r(x)=s(x)-\int_{-\infty}^{+\infty} k\left(x-x^{\prime}\right) s\left(x^{\prime}\right) \mathrm{d} x^{\prime},
$$

and the recurrent system is described as

$$
r(x)=s(x)-\int_{-\infty}^{+\infty} k\left(x-x^{\prime}\right) r\left(x^{\prime}\right) \mathrm{d} x^{\prime} .
$$

The contribution to inhibition from a neuron at position $x^{\prime}$ on the neuron at position $x$ is controlled by the weighting function $k\left(x-x^{\prime}\right)$. Equation (3) shows that lateral inhibition is modeled as a recursive feedback process; that is, the response is calculated iteratively through feedback from all neurons.

Within the limits of the Fresnel approximation the propagation of waves in free space can also be described by a linear shift-invariant model. ${ }^{3}$ And since this model applies to a large number of practical optical systems and since a Fabry-Perot cavity (FPC) can provide recursive feedback, an optical analog that displays lateral inhibition seems quite likely. In this paper I show how lateral inhibition arises naturally from the properties of coherent wave propagation, and I show specific ways in which an FPC can be incorporated into coherent optical processors. 


\section{Identification of Recurrent Lateral Inhibition in an Optical System}

Lateral inhibition can be demonstrated for free-space propagation between input Plane $P_{1}$ and output plane $\mathrm{P}_{2}$ [Fig. 1(a)]. The Fabry-Perot cavity consists of a thin slab of homogeneous media of refractive index $n$ contained between planes (or interfaces) $I_{1}$ and $I_{2}$.

The scalar diffraction of light distribution $u_{1}(x, y)$ on $\mathrm{P}_{1}$ into $u_{2}(x, y)$ on $\mathrm{P}_{2}$ is most generally described by the angular spectrum of wave formulation. From this model the source distribution is viewed as a linear combination of plane waves of amplitude $U_{1}\left(f_{x}, f_{y}\right)$ propagating with direction cosines $(\alpha, \beta, \gamma)$. The direction cosines are related to the spatial frequency as $(\alpha, \beta, \gamma)=\left[\lambda f_{x}, \lambda f_{y},\left(1-\alpha^{2}-\beta^{2}\right)^{1 / 2}\right]$, where $\lambda$ is the vacuum wavelength. The transmission of the FPC depends on the angle that each plane wave makes with the cavity. The attenuation of the wave amplitude is caused by multiple-beam interference and is determined by the distance $2 \Delta \gamma$ traveled between the interfaces, the intensity trasmission coefficient $T$, and the intensity reflection coefficient $R$ of the FPC. If there is no absorption within the FPC, then $R+T=1$.

The transfer function for a plane wave with direction cosines $(\alpha, \beta, \gamma)$ propagating in the $Z$ direction from plane $\mathrm{P}_{1}$ to a plane located at $z$ is ${ }^{3}$

$$
H\left(f_{x}, f_{y}\right)=\exp (j 2 \pi z \gamma / \lambda) .
$$

The transmission function of an FPC for a plane wave

$$
P_{1} I_{1} I_{2} \quad P_{2}
$$

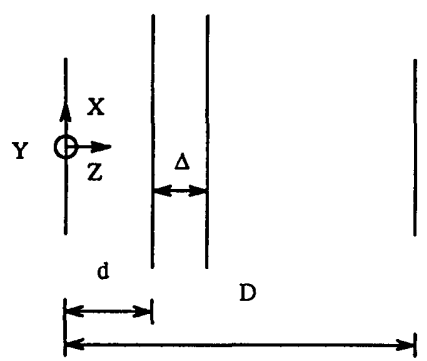

(a)

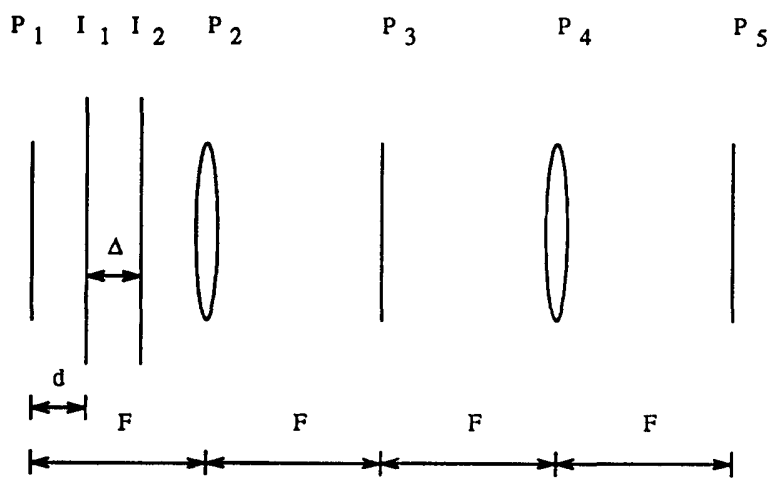

(b)

Fig. 1. (a) Geometry for optical coherent propagation in free space. The Fabry-Perot cavity with interfaces $I_{1}$ and $I_{2}$ is between $\mathrm{P}_{1}$ and $\mathrm{P}_{2}$. (b) A basic optical recurrent system. with direction cosines $(\alpha, \beta, \gamma)$ is $^{4}$

$$
T\left(f_{x}, f_{y}\right)=T /[1-R \exp (j 4 \pi n \Delta \gamma / \lambda)] .
$$

For the Fourier component $\left(f_{x}, f_{y}\right)$ on $\mathrm{P}_{2}$, its amplitude $U_{2}\left(f_{x}, f_{y}\right)$ is

$$
U_{2}\left(f_{x}, f_{y}\right)=H_{12}\left(f_{x}, f_{y}\right) T\left(f_{x}, f_{y}\right) U_{1}\left(f_{x}, f_{y}\right),
$$

where $H_{12}\left(f_{x}, f_{y}\right)$ is the transfer function of free space from $\mathrm{P}_{1}$ to $\mathrm{P}_{2}$. In Eq. (6) the value of $z$ in $H_{12}\left(f_{x}, f_{y}\right)$ is equal to $[D+(n-1) \Delta]$ rather than $D$ because of the delay difference in the cavity. The inverse Fourier transform of this result is

$$
u_{2}(x, y)=h_{12}(x, y) * t(x, y) * u_{1}(x, y),
$$

where $*$ indicates the convolution operator.

The transfer function of a one-dimensional biological recurrent system at steady state can be described mathematically as ${ }^{5}$

$$
H^{\prime}\left(f_{x}\right)=R\left(f_{x}\right) / S\left(f_{x}\right)=1 /\left[1+K\left(f_{x}\right)\right],
$$

where the capitalized variables in Eq. (8) are the Fourier transforms of the variables in Eq. (3).

Equation (5) for the FPC has the same form as Eq. (8) for recurrent lateral inhibition except for the constant scale factor $T$. Thus Eq. (7) indicates that the total response at $\mathrm{P}_{2}$ contains lateral inhibition convolved with the impulse response function $h_{12}(x, y)$.

Quite often, the field distribution is of limited angular extent. For this range the Fresnel approximation $\gamma \approx 1-\left(\alpha^{2}+\beta^{2}\right) / 2$ applies, in which case $R$ and $T$ of the FPC interfaces can reasonably be considered as constants. If we place lenses of focal lengths equal to $[D+(n-1) \Delta]$ at $\mathrm{P}_{2}$ and $\mathrm{P}_{4}$ to form a 4- $F$ system [Fig. 1(b)], $h_{12}(x, y)$ can be canceled to yield the purely recurrent process. This is done so that the frequency-domain or focal-plane response $U_{1}\left(f_{x}, f_{y}\right) T\left(f_{x}, f_{y}\right)$ is observed at the plane $\mathrm{P}_{3}$ and the lateral inhibition $u(x, y) * t(x, y)$ is observed at plane $\mathrm{P}_{5}$. The transfer function at $\mathrm{P}_{3}$ is then

$$
T\left(f_{x}, f_{y}\right)=T(r / F)=T /\{1-R \exp [j \phi(r / F)]\},
$$

where $r^{2}=x^{2}+y^{2}, f_{x}=x /(\lambda F)$, and $f_{y}=y /(\lambda F)$. The phase factor in Eq. (9) is

$$
\phi(r / F)=2 \pi m\left[1-(r / F)^{2} / 2\right],
$$

where $m=2 n \Delta / \lambda$, which is a measure of the number of wavelengths in a round trip of the cavity.

\section{Design Considerations}

The system response for practical implementations of Fig. 1(b) are band limited by apertures within the system. Usually the aperture that limits the highfrequency components is on $\mathrm{P}_{3}$. For this aperture of radius $r_{\max }$ the maximal spatial frequency $f_{\max }$ equals $r_{\max } /(\lambda F)$. We find it convenient to define the amount of runout of the phase factor in terms of a number $N_{\pi}$, where $N_{\pi} \pi=\left[\phi(0)-\phi\left(f_{\max }\right)\right]$. From Eq. (10) we 
then find

$$
r_{\max } / F=\left(N_{\pi} / m\right)^{1 / 2} .
$$

Equation (11) reflects the relations between the cavity structure, the étalon phase, and the maximal spatial frequency.

From Eqs. (9) and (10) we can see that étalon transmittance $T(r / F)$ has a minimum at $r=0$ for $m=M+1 / 2$, where $M$ is an integer, and that transmittance is comparatively enhanced for increasing $r$. This of course enhances high frequencies at $\mathrm{P}_{3}$ and improves resolution at $\mathrm{P}_{5}{ }^{3}$

Equation (5) is the result of the summation of an infinite number of reflected plane waves. It is obtained under two implicit assumptions: light is of infinite coherence length, and the FPC is of infinite lateral extent. In order to account for these nonideal limitations in real optical systems, we need to let $N$, the maximum number of wave round trips within the FPC, satisfy the approximation $R^{N+1} \approx 0$. $N$ is the integer ratio of the optical wave's coherence length to $2 n \Delta$. This condition can be met as long as the aperture of the FPC $A_{\text {FPC }}$ meets the condition

$$
\begin{aligned}
A_{\mathrm{FPC}} \geq & A_{i}+2 d \tan \theta+2(2 N+1) \\
& \times \Delta \sin \theta /\left(n^{2}-\sin ^{2} \theta\right)^{1 / 2},
\end{aligned}
$$

where $A_{i}$ denotes the aperture on the input plane $\mathrm{P}_{1}$ and $\tan \theta=r_{\max } / F$. These requirements obtain for an FPC positioned anywhere between $P_{1}$ and the lens at $\mathrm{P}_{2}$. Moving the FPC closer to the input plane (decreasing $d$ ) permits the smallest aperture for the FPC.

Lateral inhibition is maximized if we increase the refractive index of the single-slab FPC. However,

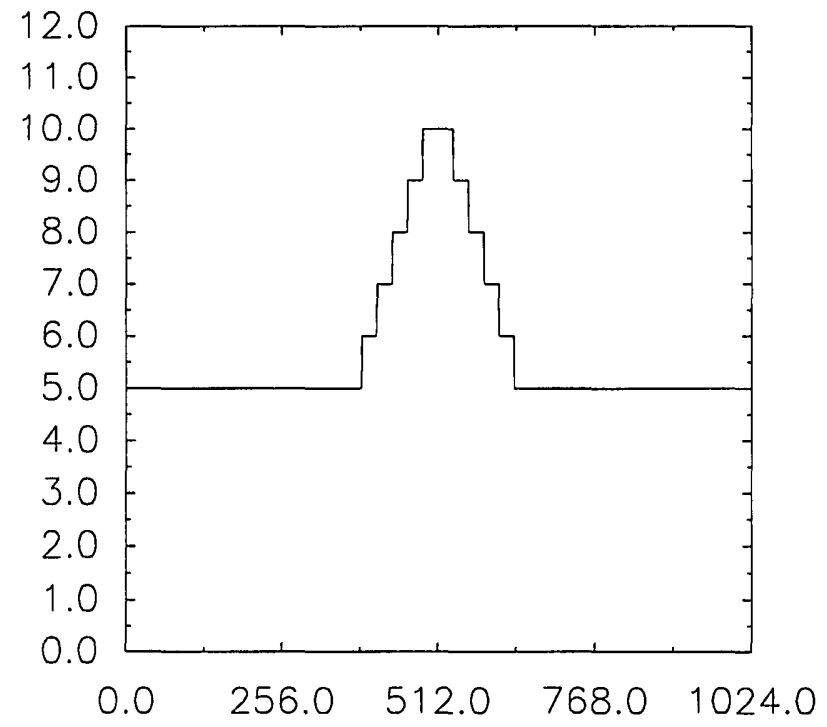

sampling point position(unit: $\lambda \vee \mathrm{m} / 2$ )

Fig. 2. Distribution of the amplitude-only object (arbitrary units) and the phase-only object (unit is $\pi / 10$ ) used in the simulations.

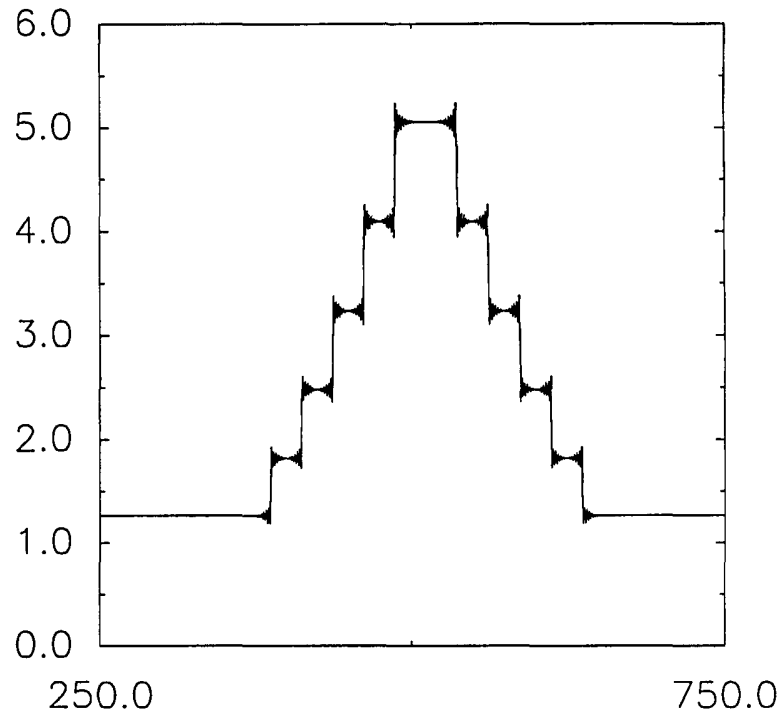

sompling point position(unit: $\lambda \sqrt{ } \mathrm{m} / 2$ )

Fig. 3. Intensity distribution (arbitrary units) of the response of $T(r / F)$ to the amplitude-only test input for $R=0.64$ and $N_{\pi}=1$.

we can also use multiple-layer cavity structure to achieve the same effect. As we see from Eq. (11), there are many physical variables that can be controlled to vary the degree of lateral inhibition. These include aperture diameter $r_{\max }$, cavity length $\Delta$, $\lambda$, and $n$, and they suggest many properties currently studied for spatial light modulation that can be used to control the degree of lateral inhibition.p4;0

\section{Computer Simulation}

We see the effect of lateral inhibition on two coherent input images with specific étalon transmittance $T(r / F)$

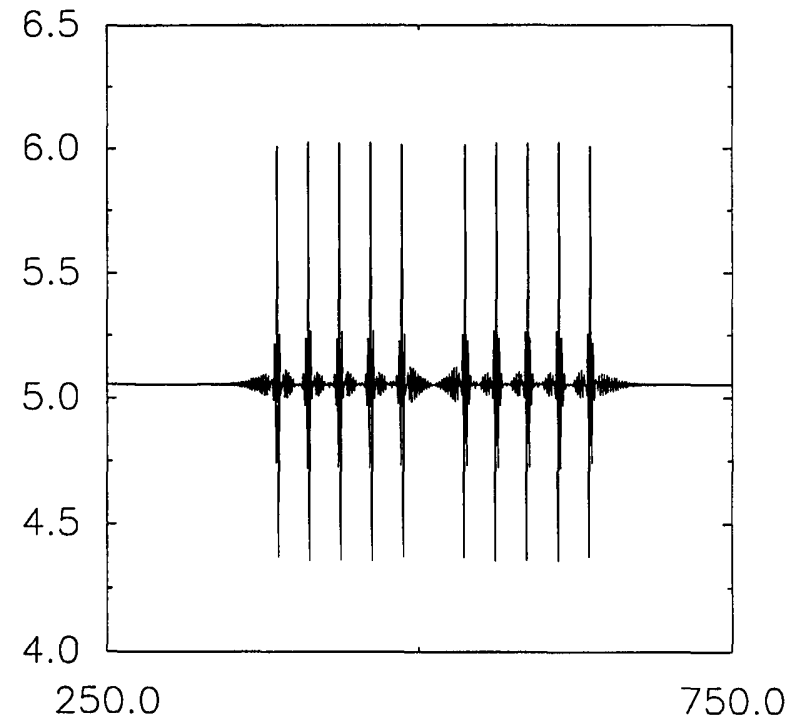

\section{sampling point position(unit: $\lambda \sqrt{ } \mathrm{m} / 2$ )}

Fig. 4. Intensity distribution (arbitrary units) of the response of $T(r / F)$ to the phase-only test input for $R=0.64$ and $N_{\pi}=1$. 
Table 1. Edge Enhancement by Optical Lateral Inhibition

\begin{tabular}{llcl}
\hline & \multicolumn{2}{c}{$\begin{array}{c}\text { Contrast for } \\
\text { Amplitude-Only Input }\end{array}$} & $\begin{array}{c}\text { Contrast for } \\
\text { Phase-Only Input }\end{array}$ \\
\hline$R$ & $C(R)$ & $C(R) / C(0)$ & $C(R)$ \\
0 & 0.44 & 1 & 0 \\
0.046 & 0.4526 & 1.0286 & 0.0148 \\
0.27 & 0.5105 & 1.1602 & 0.1011 \\
0.64 & 0.5945 & 1.3511 & 0.3245 \\
0.87 & 0.6098 & 1.3859 & 0.5982 \\
\hline
\end{tabular}

under the following conditions: $\quad m=M+1 / 2$ ( $M$ is an arbitrary integer), $N_{\pi}=1$, and $R=0.64$. One test input is an amplitude-only object and the other is a phase-only object; except for a scale factor, these are functionally identical (Fig. 2). For simulation with the fast Fourier transform I modeled each step with 25 sample points except for the central plateau, which has 50 points, and the total data file has 1024 points. The output intensity distributions are shown in Figs. 3 and 4. Figure 3 shows enhancement in the intensity at each discontinuity, and Fig. 4 shows an even more dramatic change for phase discontinuities that are not even visible in the input plane. From the viewpoint of visual perception, edges are the most important parts of an image. Therefore this new approach to spatial filtering also demonstrates the lateral inhibition of coherent images.

The enhancement of edge contrast with various values of $R$ are presented in Table 1 . Contrast $C(R)$ is defined as the ratio of the peak intensity change at the first step position to the peak intensity of the background. For $R$ equal to zero there is no inhibition, which gives contrast identical to the input images. These results show that increasing $R$ increases the edge contrast for both amplitude and phase images.

\section{Discussion and Conclusions}

I note three physical differences between a biological recurrent system and an optical recurrent system. First, in the biological recurrent system both the inhibition function and its Fourier transform are amplitude-only functions (a Gaussian or the difference of two Gaussians in the Ratliff et al. model $)^{5}$ while in the optical recurrent system the inhibition function and its Fourier transform are phase-only functions (i.e., spherical waves). Second, in the bio- logical recurrent system, signals are transported as impulses along nerve fibers, while in the optical recurrent system, signals are transported as spherical waves in free space. Third, in the biological recurrent system, negative feedback is provided by transverse interconnections among the nerve fibers, while in the optical recurrent system, negative feedback is provided by the two interfaces of the FPC. Although these two recurrent systems have three physical differences, they perform essentially identical functions.

In comparison with spatial filtering at the frequency plane, optical lateral inhibition is much less sensitive to positioning error. Spatial filters (i.e., those placed at the focal point of the object plane) are known to be sensitive to lateral offsets. ${ }^{6}$ Optical lateral inhibition uses the spatial recombination of a series of time-delayed coherent waves that are selfreferenced to the surfaces of the cavity. Thus unlike traditional spatial filters, optical lateral inhibition is insensitive to positioning errors.

I have shown that processes analogous to lateral inhibition naturally arise when a coherent image is passed through an optical system that contains a Fabry-Perot cavity. As with traditional spatial filters, it can be used to enhance image contrast including that of phase-only objects, and it can be used for optical simulation of the early visual function.

The author acknowledges support from the Scholarship of Chinese Academy of Sciences and appreciates the support of Demetri Psaltis and his group members at California Institute of Technology. I also thank Robert W. Cohn, University of Louisville, for several helpful technical discussions.

\section{References}

1. H. K. Hartline and F. Ratliff, "Inhibitory interaction of receptor units in the eye of Limulus," J. Gen. Physiol. 40, 357-376 (1957).

2. F. Ratliff, H. K. Hartline, and W. H. Miller, "Spatial and temporal aspects of retinal inhibitory interaction," J. Opt. Soc. Am. 53, 110-120 (1963).

3. J. Goodman, Introduction to Fourier Optics (McGraw-Hill, New York, 1968), Chap. 3.7, p. 48.

4. M. Born and E. Wolf, Principles of Optics, 5th ed. (Pergamon, New York, 1975), Chap. 7.6, p. 323

5. F. Ratliff, B. W. Knight, and N. Graham, "On tuning and amplification by lateral inhibition," Proc. Natl. Acad. Sci. (USA) 62, 737-740 (1969).

6. A. VanderLugt, Optical Signal Processing (Wiley, New York, 1992), Chap. 6.8, p. 279. 